\title{
Does multiparametric US improve diagnostic accuracy in the characterization of small testicular masses?
}

\author{
Alfonso Reginelli ${ }^{1}$, Alfredo D'Andrea ${ }^{1}$, Alfredo Clemente ${ }^{1}$, Andrea Izzo $^{1}$, Fabrizio Urraro ${ }^{1}$, \\ Fernando Scala ${ }^{1}$, Valerio Nardone ${ }^{2}$, Cesare Guida ${ }^{2}$, Michele Scialpi $^{3}$, Salvatore Cappabianca ${ }^{1}$ \\ ${ }^{1}$ Radiology and Radiotherapy Unit, Department of Precision Medicine, University of Campania "L. Vanvitelli”, Naples, Italy; ${ }^{2}$ Unit of Radiation \\ Oncology, Ospedale del Mare, Naples, Italy; ${ }^{3}$ Section of Diagnostic Imaging, Department of Surgical and Biomedical Sciences, S. Maria della \\ Misericordia Hospital, Perugia University, S. Andrea delle Fratte, Perugia, Italy \\ Contributions: (I) Conception and design: A Reginelli, A D'Andrea, A Clemente, A Izzo, M Scialpi, S Cappabianca; (II) Administrative support: A \\ Reginelli, F Urraro, FS, VN, CG, M Scialpi, S Cappabianca; (III) Provision of study materials or patients: A Reginelli, A Clemente, A Izzo, F Scala, \\ V Nardone, C Guida; (IV) Collection and assembly of data: A D’Andrea, A Clemente, A Izzo, F Urraro, M Scialpi, S Cappabianca; (V) Data analysis \\ and interpretation: A Reginelli, A D'Andrea, A Clemente, A Izzo, M Scialpi; (VI) Manuscript writing: All authors; (VII) Final approval of manuscript: \\ All authors. \\ Correspondence to: Alfonso Reginelli, MD. Radiology and Radiotherapy Unit, Department of Precision Medicine, University of Campania "L. \\ Vanvitelli, Piazza Miraglia, 2, 80138 Naples, Italy. Email: alfonsoreginelli@hotmail.com.
}

Background: Recent advances in ultrasonography (US) have produced new innovative techniques for the non-invasive assessment of testicular masses. The aim of this study was to investigate the diagnostic performance of multiparametric US, including gray-scale, Color-power Doppler and real-time elastography (RTE) analysis, in the characterization of testicular lesions.

Methods: Fifty-four patients (median 42.2 years; range, 10-64 years) with testicular lesions detected with gray-scale US and power Doppler US were evaluated with RTE. The tissue elasticity was assessed in all lesions. Hard lesions were suspected of being malignant while testicular lesions with normal or decreased tissue stiffness (soft lesions) were considered benign. Intraoperative findings were the standard of reference. Sensitivity, specificity, negative predictive value, positive predictive value, and diagnostic accuracy were calculated for each US method and in combination.

Results: Forty-six of the 54 lesions (85.2\%) were testicular malignant tumors. Thirty-five out of 46 (76\%) were $\geq 2 \mathrm{~cm}$ [seminomas ( $\mathrm{n}=18)$, mixed seminomatous and/or nonseminomatous tumors $(\mathrm{n}=9)$, embryonal carcinomas $(n=2)$, immature teratomas $(n=3)$ and Leydig cell tumors $(n=3)]$ while the remaining 11 tumors were $<2 \mathrm{~cm}$ [seminomas $(\mathrm{n}=5)$, mixed germinal cells tumors $(\mathrm{n}=2)$, immature teratomas $(\mathrm{n}=2)$ and Leydig cell tumors $(n=2)$ ]. Eight out of 54 lesions $(14.8 \%)$ were benign lesions (orchitis $n=2$, dermoid cyst $n=1$, adrenal rest $n=1$, papillary cystadenoma $n=1$, sclero-hyaline nodule $n=1$, focal fibrosis $n=1$ and post-traumatic focal fibrosis $n=1$ ). RTE showed the presence of hard pattern in 40 out of 46 (87\%) malignant tumors and in 2 out of 8 (25\%) of benign lesions. The combination of gray-scale US, Color-power Doppler and RTE aided a sensitivity of $100 \%$, a specificity of $83 \%$, a negative predictive value of $100 \%$, a positive predictive value of $91 \%$ and accuracy of $90 \%$.

Conclusions: RTE demonstrated to increase the diagnostic accuracy of conventional US in the characterization of testicular lesions providing additional information on tissue stiffness. The multiparametric US evaluation has proven to increase the diagnostic performance in the characterization of testicular lesions.

Keywords: Ultrasound; sonoelastography; testis; scrotum

Submitted Jan 29, 2019. Accepted for publication Apr 02, 2019.

doi: 10.21037 /gs.2019.04.03

View this article at: http://dx.doi.org/10.21037/gs.2019.04.03

(c) Gland Surgery. All rights reserved. 


\section{Introduction}

Testicular cancers represent $1 \%$ of all male cancers (1). Most of testicular tumors occur in young men between 18-35 years and seminomatous forms represent the most common among all histological subtypes (2). Radiologist plays a pivotal role in identifying tumor, staging disease, and managing the followup imaging surveillance (3). Moreover, a non-invasive lesion characterization is mandatory for an appropriate treatment (radical orchiectomy or testis-sparing) or, conversely, could potentially help avoiding unnecessary testicular surgery (4-6). Among all imaging modalities, the scrotal ultrasonography (US) is the first level imaging technique in the evaluation of testicular masses (5). The combination of gray-scale and Color-power Doppler US has demonstrated a high sensitivity $(100 \%)$ in the detection of testicular lesions $(4,7)$. Nevertheless, these techniques do not consent a definitive differentiation of malignancies from testicular benign conditions, such as orchitis, dermoid cyst, granuloma, focal fibrosis, adrenal rest and papillary cystadenoma (8-10). In fact, those lesions can mimic cancer and as consequence the specificity of gray-scale and Color-power Doppler US is lower than sensitivity (11-19). Therefore, an imaging technique able to increase the specificity of gray-scale and Color-power Doppler US in the assessment of testicular lesions, could improve the diagnostic performance of this imaging modality (20-26). Real-time elastography (RTE) is a modern US method able to assess the tissue elasticity with many proven benefits in the evaluation of malignant tumors (20). In particular, most of cancers show an increased tissue stiffness in relation with their higher cell density compared with the surrounding normal tissues (20,27-31). This would be an additional information that can increase the specificity and diagnostic accuracy of US technique in the assessment of testicular lesions.

The aim of this study was to investigate the diagnostic performance of multiparametric US, including grayscale US, Color-power Doppler and RTE analysis, in the characterization of testicular lesions.

\section{Methods}

This retrospective study obtained the ethical approval by the institutional review board and the requirement written informed consent was obtained from all patients.

\section{Patient population}

Fifty-four consecutive patients (median 42.2 years; range,
10-64 years) with clinical suspicion of testicular mass, underwent US examinations including gray-scale US, Color-power Doppler and RTE analysis from February 2012 to April 2014. All testicular lesions with pathological confirmation were included in this study. Patient data, clinical markers ( $\alpha$-fetoprotein and $\beta$-human-chorionic gonadotropin), histopathology and US reports were retrospectively analyzed.

\section{Imaging analysis}

All gray-scale US, Color-power Doppler US and RTE were performed by 15 years experienced uroradiologist using a Hi-Vision Preirus unit (Hitachi Medical Systems, Tokyo, Japan) with a 5.0-13.0 MHz linear probe. Colorpower Doppler US scans were set at $10 \mathrm{MHz}$ frequency with pulse repetition frequency of $16 \mathrm{~cm} / \mathrm{sec}$, frame rate of 12 pictures per second. RTE evaluations were performed through the multi-compression of the scrotum in order to improve the signal-to-noise ratio. The compression was adjusted according to the visual indicator for compression on the US system monitor. Calculation of tissue elasticity was in real time (up to 30 frames per second), and stiffness of testicular tissue was displayed with a color overlay on the B-mode images. For each lesion, axial and transverse scans were performed according to Dogra et al. (4). At gray-scale US, lesion size (expressed in $\mathrm{mm}$ in 3 planes), homogeneity, echogenicity (hypo-, iso- and hyper-echoic to the surrounding parenchyma) and pseudo-capsule appearance were analyzed. At Color-power Doppler US, the vascular signals (hypo-, iso- or hyper- compared with normal testicular tissue) were documented. The RTE tissue stiffness was encoded from red (soft) to blue (hard) according to the standard settings of the US system. The evaluations on tissue elasticity were reported in a chromatic value and categorized in three patterns: (I) soft (red); (II) medium (green): and (III) hard (blue). For each patient, representative images were recorded on external storage system.

\section{Reference standard}

All lesions $\geq 2 \mathrm{~cm}$ underwent surgical exploration and histopathologic results were correlated with imaging findings. All nodules $<2 \mathrm{~cm}$ with malignant US pattern underwent inguinoscrotal exploration followed by nodulectomy. The nodules $<2 \mathrm{~cm}$ without malignant US appearances, such as homogeneous pattern at gray scale US, absence of intrinsic vascularization at Color-power Doppler US and soft- 
medium pattern at RTE analysis, were followed over time.

\section{Statistical analysis}

Sensitivity, specificity, positive and negative predictive values and diagnostic accuracy were calculated for grayscale, Color-power Doppler US and RTE alone and in combination. The Chi-squared test was used to compare the diagnostic accuracy of different US techniques in the diagnosis of malignant from benign lesions. A twotailed $\mathrm{P}$ value of less than 0.05 was considered to indicate a statistically significant difference. All statistical analyses were performed using commercially available software (SPSS, version 11, SPSS, Chicago, IL, USA).

\section{Results}

All lesions ( $\mathrm{n}=54)$ were categorized according to their size: lesions $\geq 2 \mathrm{~cm} \mathrm{(n=35)}$ and $<2 \mathrm{~cm}(\mathrm{n}=19)$. All lesions $\geq 2 \mathrm{~cm}$ underwent radical orchifunicolectomy and histopathologic examination confirmed their malignancy: seminomas $(\mathrm{n}=18)$, mixed seminomatous and/or non-seminomatous tumors $(n=9)$, embryonal carcinomas $(n=2)$, immature teratomas $(n=3)$ and Leydig cell tumors $(n=3)$. Alterations of serum markers ( $\alpha$-FP, $\beta$-HCG) were found in all patients with malignancy. The results of gray-scale, Color-power Doppler and RTE analysis of the 35 lesions $\geq 2 \mathrm{~cm}$, are reported in Table S1. A significant statistical correlation between the gray-scale US findings and the tumor type (seminoma vs. non-seminoma) were found $(\mathrm{P}<0.001)$. No statistical differences between Color-power Doppler US and RTE findings was observed for seminomatous from non-seminomatous tumors.

All patients $(\mathrm{n}=19)$ with testicular lesion $<2 \mathrm{~cm}$ showed undefined alterations of serum markers. The results of gray-scale, Color-power Doppler and RTE analysis of the 19 lesions $<2 \mathrm{~cm}$, are reported in Table S2. Eleven out of 19 patients $(58 \%)$ showed US malignant features such as non-homogeneous pattern at gray-scale, intrinsic vascularization at Color-power Doppler or hard pattern at RTE (Figures 1,2) and underwent inguinoscrotal exploration followed by surgical nodulectomy. After the extemporaneous examination, all malignancies underwent radical orchifunicolectomy. Histopathologic examination revealed: seminomas $(n=5)$, mixed germinal cells tumors $(\mathrm{n}=2)$, immature teratomas $(\mathrm{n}=2)$ and Leydig cell tumors $(\mathrm{n}=2)$. The remaining 8 out of 19 patients $(42 \%)$ with lesions $<2 \mathrm{~cm}$ showed US benign features such as solid homogeneous pattern at gray-scale, absence of intrinsic vascularization at Color-power Doppler and soft-medium pattern at RTE. Two of them underwent extemporaneous biopsy (sclero-ialine nodule $\mathrm{n}=1$ and post-traumatic focal fibrosis $n=1$ ) while the remaining 6 of them were re-evaluated by US examination over the time showing stability in size, echogenicity, vascularization and elasticity. The extemporaneous biopsy performed in the latter group of patients revealed: focal fibrosis $n=1$, orchitis $n=2$, dermoid cyst $\mathrm{n}=1$, adrenal rest $\mathrm{n}=1$ and papillary cystadenoma $\mathrm{n}=1$.

The conventional US including gray-scale and Colorpower Doppler evaluation aided a sensitivity of $86 \%$, a specificity of $64 \%$, a negative predictive value of $48 \%$, a positive predictive value of $84 \%$, and accuracy of $81 \%$ for testicular tumor diagnosis. Statistical analysis showed a sensitivity of $85 \%$, a specificity of $78 \%$, a negative predictive value of $71 \%$, a positive predictive value of $93 \%$ and an accuracy of $87 \%$ for RTE in testicular tumor diagnosis. The combination of gray-scale US, Color-power Doppler and RTE aided a sensitivity of $100 \%$, a specificity of $83 \%$, a negative predictive value of $100 \%$, a positive predictive value of $91 \%$ and accuracy of $90 \%$.

\section{Discussion}

The differentiation between benign and malignant testicular masses is essential in order to establish an appropriate treatment or a surveillance protocol. Grayscale US is the first line diagnostic examination able to depict testicular tumors in most cases. However, the differential diagnosis between benign and malignant tumors remains difficult basing only on gray-scale and Colorpower Doppler US, especially for small lesions $<2 \mathrm{~cm}(4,5)$. In many cases, the conventional US examination is able to determine the nature of the lesion with high accuracy. For example, testicular cystic masses are very easy to characterize due to their fluid content without any further diagnostic integration. Otherwise, many focal lesions could present indeterminate US features which does not allow an unequivocal diagnosis. Magnetic resonance imaging has been proposed as an additional imaging technique to conventional US in cases of uncertainty, but it remains an expensive method with limited availability (32-35). In order to increase the diagnostic value of conventional US in differentiating malignant from benign lesions, some Authors has recently reported many advantages derived from the tissue elasticity analysis performed by RTE $(20,36)$. In general, the most of malignant lesions show an increased stiffness because they have higher cell density, if 


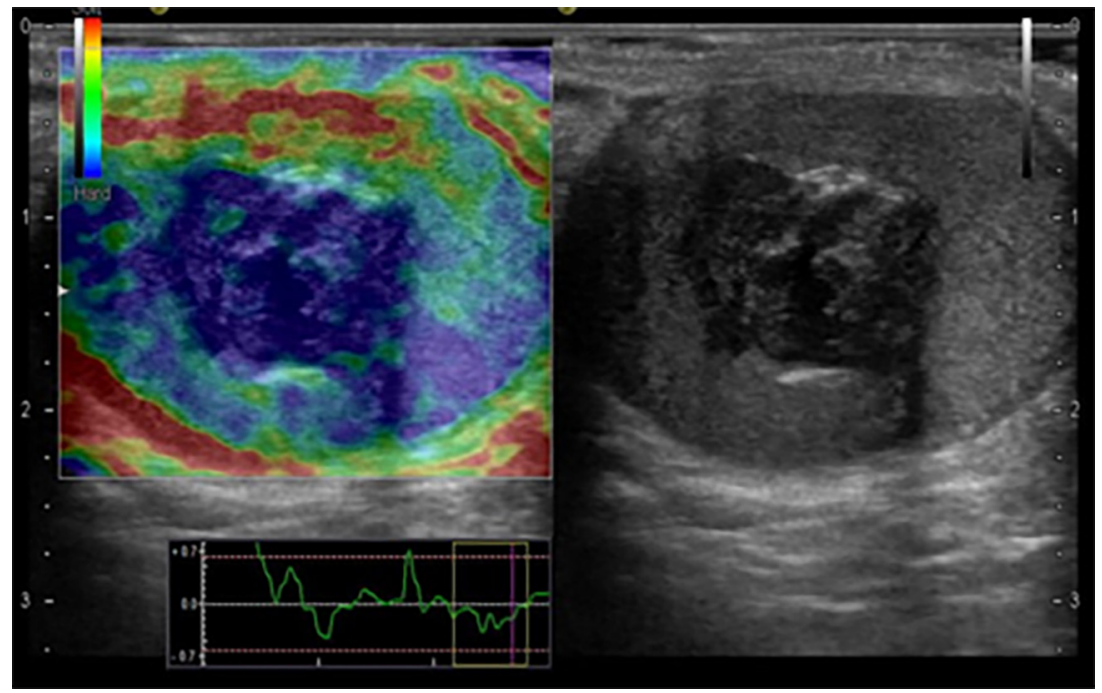

Figure 1 A 32-year-old man with a seminoma. Image from RTE shows a hard lesion (blue) while gray-scale US scan shows a hypoechoic mass. RTE, real-time elastography; US, ultrasonography.

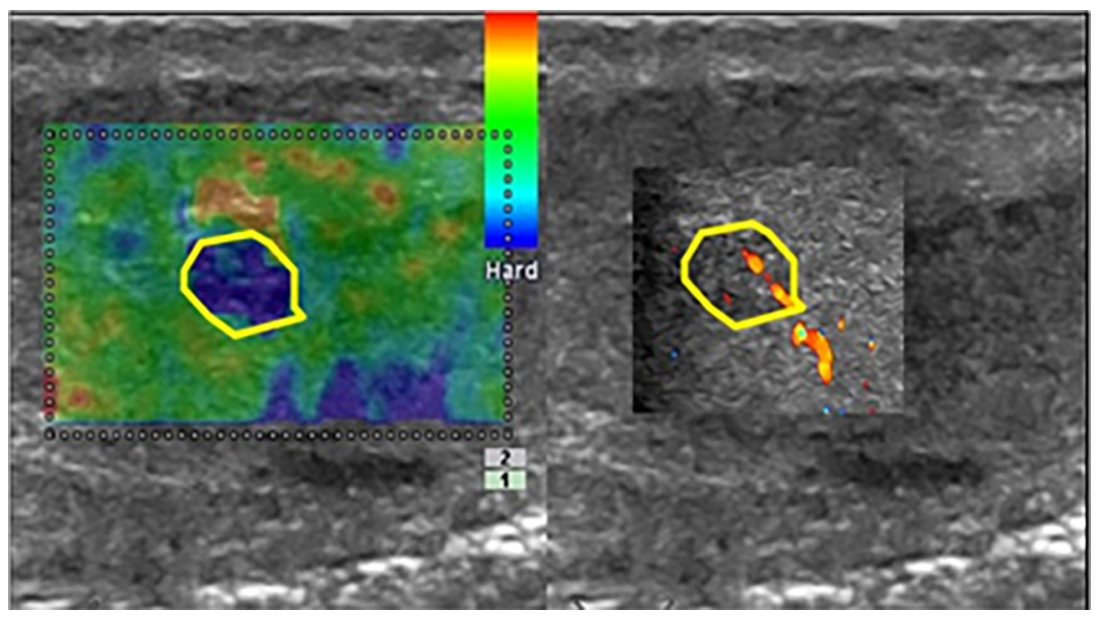

Figure 2 A 42-year-old man with a small hypoechoic oval mass in the right testicle with hard pattern from the RTE analysis and showing intralesional vascularization at Color-power-Doppler image. Histology confirmed a sub-centimetric seminoma in cryptorchid testis. RTE, real-time elastography.

compared with the surrounding normal tissues. Our results showed a notable prevalence of the hard "blue" pattern in testicular malignancies (40 out of $46 ; 87 \%$ ). These results are in line with some previous experiences, in which the Authors documented an increase of elasticity in the most of testicular cancers $(4,20)$. However, according to Aigner et al. (20), some benign testicular lesions are commonly associated with an increased stiffness at RTE evaluation, referable to fibrosis, central scar, orchitis or adrenal rest. In our study, RTE evaluation showed a hard pattern in 2 out of $8(25 \%)$ benign lesions (all $<2 \mathrm{~cm}$ ) and in only 5 out of 46 cases $(11 \%)$ with mixed pattern was found malignant. Excepting the 5 malignant lesions (5 teratomas) with a soft-medium pattern, all malignant lesions showed a tissue "hard" pattern, underlining the extremely high sensitivity of RTE. The low stiffness of teratomas was likely a result of the high tissues inhomogeneity and due to the high presence adipocytes, as also previously reported by some Authors (20,30). Furthermore, we found two benign lesions with a hard pattern on RTE evaluation (1 focal orchitis 
and 1 hyaline nodule) that were distinguished, thanks to the absence of signal-color on Color-power Doppler US. As reported in literature (4,20,30), Leydig cell tumors can demonstrate a variable pattern on RTE, depending on the number and size of Leydig cells, lymphatic or vascular invasion. In our experience, the 5 Leydig cell tumors showed a hard-stiff pattern reflecting their high cell and vessel density confirmed after surgery.

Our experience demonstrated a clear potentiality of RTE in improving the sensitivity and diagnostic accuracy of the US examination (gray-scale and Color-power Doppler US) respectively up to $100 \%$ and $90 \%$. These results are in line with the few previous published series, reporting a comparable sensitivity and diagnostic accuracy, 100\% and $92 \%$ respectively $(4,20)$. Moreover, in our study the multiparametric US analysis has demonstrated a greater specificity (83\%) when compared to single US analysis. However, our study has a number of limitations. First, the retrospective study population was relatively small $(n=54)$, although we have considered various types of malignant and benign lesions. Finally, RTE was performed with only one US system and our results may be different from results obtained by another US system such as the different operator.

In conclusion, RTE has the potential to increase the diagnostic accuracy of gray-scale and Color-power Doppler US in the testicular lesions' evaluation. The multiparametric US evaluation has proven to increase the diagnostic performance in the characterization of testicular lesions, especially for those $<2 \mathrm{~cm}$, avoiding unnecessary surgery especially in young patients.

\section{Acknowledgments}

None.

\section{Footnote}

Conflicts of Interest: The authors have no conflicts of interest to declare.

Ethical Statement: This retrospective study obtained the ethical approval by the institutional review board and the requirement written informed consent was obtained from all patients.

\section{References}

1. Purdue MP, Devesa SS, Sigurdson AJ, et al. International patterns and trends in testis cancer incidence. Int J Cancer 2005;115:822-7.

2. Ulbright TM. Germ cell tumors of the gonads: a selective review emphasizing problems in differential diagnosis, newly appreciated, and controversial issues. Mod Pathol 2005;18:S61-79.

3. Coursey Moreno C, Small WC, Camacho JC, et al. Testicular tumors: what radiologists need to knowdifferential diagnosis, staging, and management. Radiographics 2015;35:400-15.

4. Dogra VS, Gottlieb RH, Oka M, et al. Sonography of the scrotum. Radiology 2003;227:18-36.

5. Chini MG, Riccio R, Bifulco G. DFT/NMR integrated approach: a valid support to the total synthesis of chiral molecules. Magn Reson Chem 2008;46:962-8.

6. Hamm B. Differential diagnosis of scrotal masses by ultrasound. Eur Radiol 1997;7:668-79.

7. Russo S, Lo Re G, Galia M, et al. Videofluorography swallow study of patients with systemic sclerosis. Radiol Med 2009;114:948-59.

8. De Bernardi IC, Floridi C, Muollo A, et al. Vascular and interventional radiology radiofrequency ablation of benign thyroid nodules and recurrent thyroid cancers: literature review. Radiol Med 2014;119:512-20.

9. Tamburrini S, Solazzo A, Sagnelli A, et al. Amyotrophic lateral sclerosis: sonographic evaluation of dysphagia. Radiol Med 2010;115:784-93.

10. Yagil Y, Naroditsky I, Milhem J, et al. Role of Doppler ultrasonography in the triage of acute scrotum in the emergency department. J Ultrasound Med 2010;29:11-21.

11. Elzinga-Tinke JE, Sirre ME, Looijenga LH, et al. The predictive value of testicular ultrasound abnormalities for carcinoma in situ of the testis in men at risk for testicular cancer. Int J Androl 2010;33:597-603.

12. Lenz S. Cancer of the testicle diagnosed by ultrasound and the ultrasonic appearance of the contralateral testicle. Scand J Urol Nephrol Suppl 1991;137:135-8.

13. Mandato Y, Reginelli A, Galasso R, et al. Errors in the radiological evaluation of the alimentary tract: part $\mathrm{I}$. Semin Ultrasound CT MR 2012;33:300-7.

14. De Filippo M, Gira F, Corradi D, et al. Benefits of $3 \mathrm{D}$ technique in guiding percutaneous retroperitoneal biopsies. Radiol Med 2011;116:407-16.

15. Kreydin EI, Barrisford GW, Feldman AS, et al. Testicular cancer: what the radiologist needs to know. AJR Am J Roentgenol 2013;200:1215-25.

16. Cappabianca S, Iaselli F, Reginelli A, et al. Value of diffusion-weighted magnetic resonance imaging in the 
characterization of complex adnexal masses. Tumori 2013;99:210-7.

17. Palma BD, Guasco D, Pedrazzoni M, et al. Osteolytic lesions, cytogenetic features and bone marrow levels of cytokines and chemokines in multiple myeloma patients: Role of chemokine (C-C motif) ligand 20. Leukemia 2016;30:409-16.

18. Strocchia M, Terracciano S, Chini MG, et al. Targeting the Hsp90 C-terminal domain by the chemically accessible dihydropyrimidinone scaffold. Chem Commun (Camb) 2015;51:3850-3.

19. D'Andrea A, Coppolino F, Cesarano E, et al. US in the assessment of acute scrotum. Crit Ultrasound J 2013;5 Suppl 1:S8.

20. Aigner F, De Zordo T, Pallwein-Prettner L, et al. Realtime sonoelastography for the evaluation of testicular lesions. Radiology 2012;263:584-9.

21. Cappabianca S, Reginelli A, Monaco L, et al. Combined videofluoroscopy and manometry in the diagnosis of oropharyngeal dysphagia: examination technique and preliminary experience. Radiol Med 2008;113:923-40.

22. Zoccali C, Rossi B, Zoccali G, et al. A new technique for biopsy of soft tissue neoplasms: A preliminary experience using MRI to evaluate bleeding. Minerva Medica 2015;106:117-20.

23. Cappabianca S, Porto A, Petrillo M, et al. Preliminary study on the correlation between grading and histology of solitary pulmonary nodules and contrast enhancement and $[18 \mathrm{~F}]$ fluorodeoxyglucose standardised uptake value after evaluation by dynamic multiphase CT and PET/CT. J Clin Pathol 2011;64:114-9.

24. Golfieri R, Garzillo G, Ascanio S, et al. Focal lesions in the cirrhotic liver: Their pivotal role in gadoxetic acidenhanced MRI and recognition by the Western guidelines. Dig Dis 2014;32:696-704.

25. Vasuri F, Golfieri R, Fiorentino M, et al. OATP 1B1/1B3 expression in hepatocellular carcinomas treated with orthotopic liver transplantation. Virchows Archiv 2011;459:141-6.

26. Regini E, Bagnera S, Tota D, et al. Role of sonoelastography in characterising breast nodules: preliminary experience with 120 lesions. Radiol Med 2010;115:551-62.

27. Krouskop TA, Wheeler TM, Kallel F, et al. Elastic moduli of breast and prostate tissues under compression. Ultrason Imaging 1998;20:260-74.
28. Reginelli A, Clemente A, Cardone C, et al. Computed tomography densitometric study of anti-angiogenic effect of regorafenib in colorectal cancer liver metastasis. Future Oncol 2018;14:2905-13.

29. Renzulli M, Clemente A, Brocchi S, et al. LI-RADS: a great opportunity not to be missed. Eur J Gastroenterol Hepatol 2019;31:283-8.

30. Tovoli F, Renzulli M, Negrini G, et al. Inter-operator variability and source of errors in tumour response assessment for hepatocellular carcinoma treated with sorafenib. Eur Radiol 2018;28:3611-20.

31. Compagnone G, Giampalma E, Domenichelli S, et al. Calculation of conversion factors for effective dose for various interventional radiology procedures. Med Phys 2012;39:2491-8.

32. Mohrs OK, Thoms H, Egner T, et al. MRI of patients with suspected scrotal or testicular lesions: diagnostic value in daily practice. AJR Am J Roentgenol 2012;199:609-15.

33. Ferrari F, Arrigoni F, Miccoli A, et al. Effectiveness of Magnetic Resonance-guided Focused Ultrasound Surgery (MRgFUS) in the uterine adenomyosis treatment: technical approach and MRI evaluation. Radiologia Medica 2016;121:153-61.

34. Carotti M, Galeazzi V, Catucci F, et al. Clinical utility of eco-color-power doppler ultrasonography and contrast enhanced magnetic resonance imaging for interpretation and quantification of joint synovitis: A review. Acta Biomedica 2018;89:48-77.

35. Mascaretti S, Ferrari F, Miccoli A, et al. Feasibility of magnetic resonance-guided focus ultrasound surgery (MRgFUS) in the uterine fibroid treatment: Evaluation of the treatment radicalization in single and multiple fibroids correlated to clinical outcome. Clin Exp Obstet Gynecol 2018;45:84-7.

36. Goddi A, Sacchi A, Magistretti G, et al. Real-time tissue elastography for testicular lesion assessment. Eur Radiol 2012;22:721-30.

Cite this article as: Reginelli A, D'Andrea A, Clemente A, Izzo A, Urraro F, Scala F, Nardone V, Guida C, Scialpi M, Cappabianca S. Does multiparametric US improve diagnostic accuracy in the characterization of small testicular masses? Gland Surg 2019;8(Suppl 3):S136-S141. doi: 10.21037/ gs.2019.04.03 
Table S1 Gray scale, power Doppler US and RTE findings of 35 lesions $\geq 2 \mathrm{~cm}$

\begin{tabular}{|c|c|c|c|c|c|c|c|c|c|c|c|c|}
\hline \multirow{2}{*}{$\begin{array}{l}\text { Lesion type } \\
\text { (maximum diameter } \geq 2 \mathrm{~cm} \text { ) }\end{array}$} & \multirow[b]{2}{*}{$\mathrm{N}$} & \multicolumn{5}{|c|}{ US pattern } & \multicolumn{3}{|c|}{ Color Doppler pattern } & \multicolumn{3}{|c|}{ RTE pattern } \\
\hline & & Homogeneous & Inhomogeneous & Hypo-echoic & $\begin{array}{l}\text { Mixed echogenicity } \\
\text { /isoechoic }\end{array}$ & Hyper-echoic & Peripheral & Intralesional & $\begin{array}{c}\text { Mixed } \\
\text { vascularization }\end{array}$ & Hard blue & Green & $\begin{array}{l}\text { Red-green-blue } \\
\text { (mixed) }\end{array}$ \\
\hline Seminomas & 18 & 18 & - & 10 & 8 & - & - & 5 & 13 & 18 & - & - \\
\hline $\begin{array}{l}\text { Mixed seminomatous and/or } \\
\text { nonseminomatous tumors }\end{array}$ & 9 & 5 & 4 & 8 & 1 & - & 5 & 2 & 2 & 9 & - & - \\
\hline Embryonal carcinoma & 2 & - & 2 & 1 & 1 & - & - & 2 & - & 2 & - & - \\
\hline Immature teratoma & 3 & - & 3 & 1 & 1 & 1 & - & 3 & - & - & 1 & 2 \\
\hline Leydig cell tumors & 3 & 2 & 1 & - & - & 3 & 2 & - & 1 & 3 & - & - \\
\hline Total & 35 & 25 & 10 & 20 & 11 & 4 & 7 & 12 & 16 & 32 & 1 & 2 \\
\hline
\end{tabular}

RTE, real-time elastosonography; US, ultrasonography.

Table S2 Gray scale, Color-power Doppler US and RTE findings of 19 lesions $<2 \mathrm{~cm}$

\begin{tabular}{|c|c|c|c|c|c|c|c|c|c|c|c|c|}
\hline \multirow{2}{*}{$\begin{array}{l}\text { Lesion type } \\
\text { (maximum diameter }<2 \mathrm{~cm} \text { ) }\end{array}$} & \multirow[b]{2}{*}{$\mathrm{N}$} & \multicolumn{5}{|c|}{ US pattern } & \multicolumn{3}{|c|}{ Color Doppler pattern } & \multicolumn{3}{|c|}{ RTE pattern } \\
\hline & & Homogeneous & Inhomogeneous & Hypo-echoic & $\begin{array}{l}\text { Mixed echogenicity } \\
\text { /isoechoic }\end{array}$ & Hyper-echoic & Peripheral & Intralesional & $\begin{array}{c}\text { Mixed } \\
\text { vascularization }\end{array}$ & Hard blue & Green & $\begin{array}{l}\text { Red-green-blue } \\
\text { (mixed) }\end{array}$ \\
\hline Seminomas & 5 & 5 & - & 4 & 1 & - & - & - & 5 & 5 & - & - \\
\hline $\begin{array}{l}\text { Mixed seminomatous and/or } \\
\text { nonseminomatous tumors }\end{array}$ & 2 & 2 & - & 2 & - & - & - & 1 & 1 & 2 & - & - \\
\hline Immature Teratoma & 2 & 1 & 1 & 1 & 1 & - & - & - & 2 & - & - & 2 \\
\hline Leydig cell tumors & 2 & 1 & 1 & - & 1 & 1 & 2 & - & - & 2 & - & - \\
\hline Orchitis & 2 & - & 2 & 1 & - & 1 & 1 & - & 1 & 1 & - & 1 \\
\hline Dermoid cyst & 1 & - & 1 & - & - & 1 & 1 & - & - & - & 1 & - \\
\hline Adnexal residual & 1 & - & 1 & - & - & 1 & - & - & 1 & - & 1 & - \\
\hline Papillary cystadenoma & 1 & - & 1 & - & 1 & - & 1 & - & - & - & - & 1 \\
\hline Sclero-hyaline nodule & 1 & 1 & - & 1 & - & - & 1 & - & - & 1 & - & - \\
\hline Focal fibrosis & 1 & 1 & - & 1 & - & - & 1 & - & - & - & 1 & - \\
\hline Post-traumatic focal fibrosis & 1 & 1 & - & 1 & - & - & 1 & - & - & - & 1 & - \\
\hline Total & 19 & 12 & 7 & 11 & 4 & 4 & 8 & 1 & 10 & 11 & 4 & 4 \\
\hline
\end{tabular}

RTE, real-time elastosonography; US, ultrasonography. 